
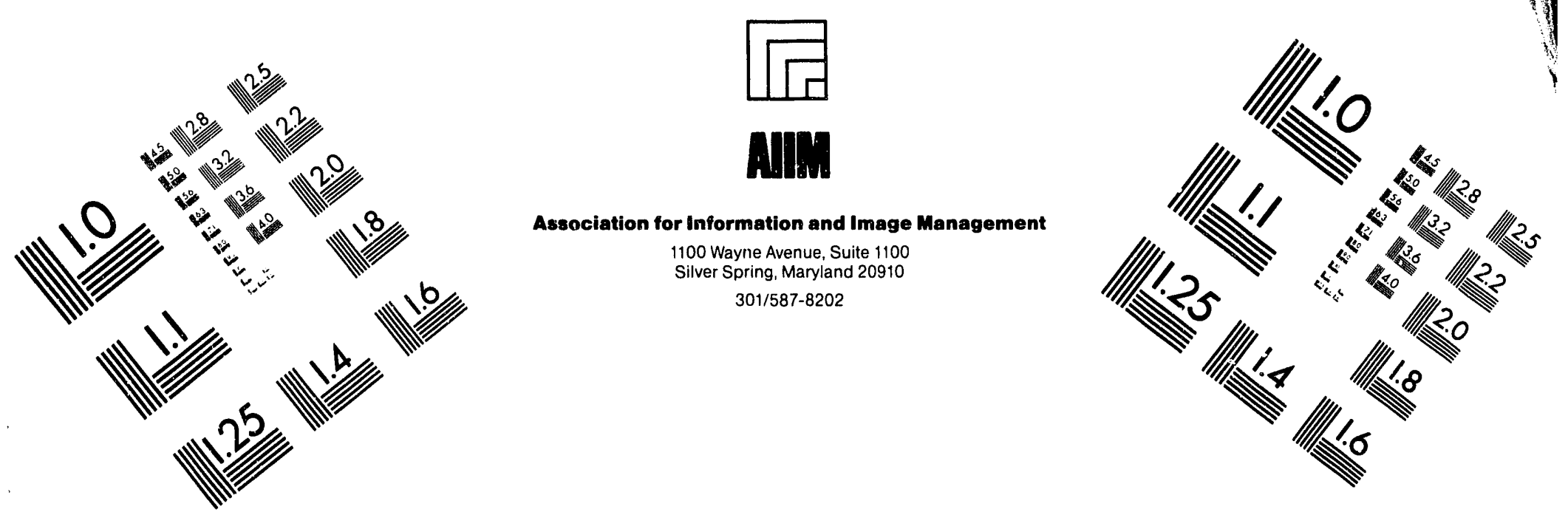

\title{
Centimeter
}

$\begin{array}{lllllllllllllll}1 & 2 & 3 & 4 & 5 & 6 & 7 & 8 & 9 & 10 & 11 & 12 & 13 & 14 & 15 \\ \mathrm{~mm}\end{array}$ $\mid$ Inches
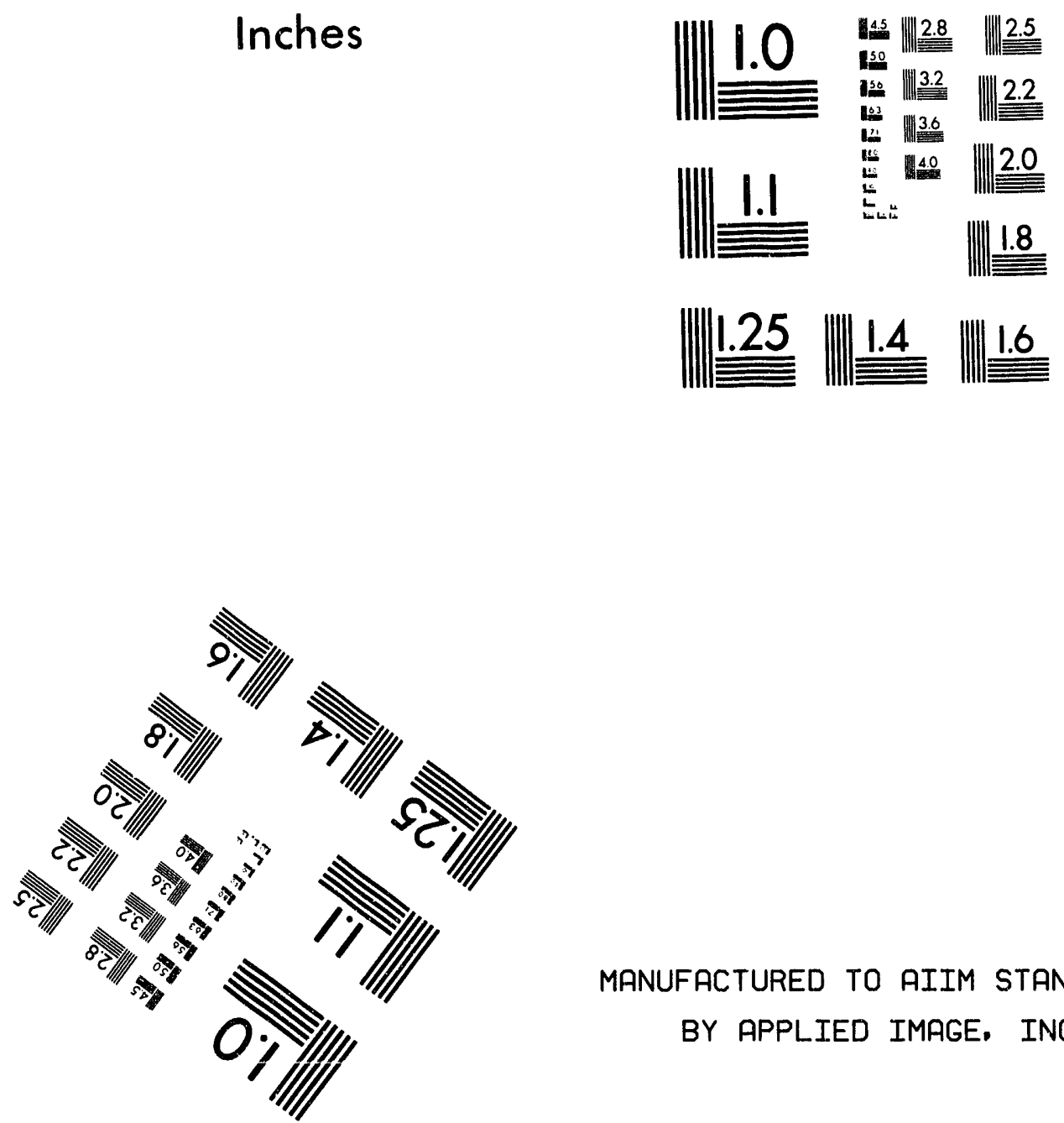

MANUFACTURED TO AIIM STANDARDS

BY APPLIED IMAGE, INC.

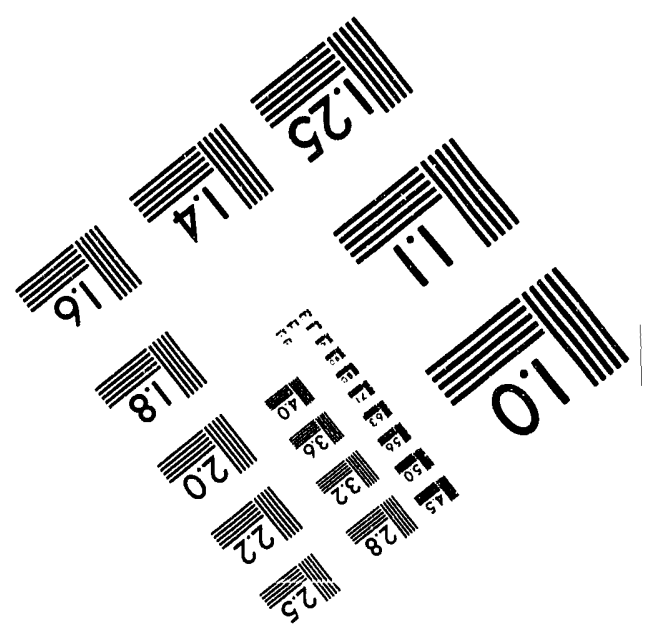



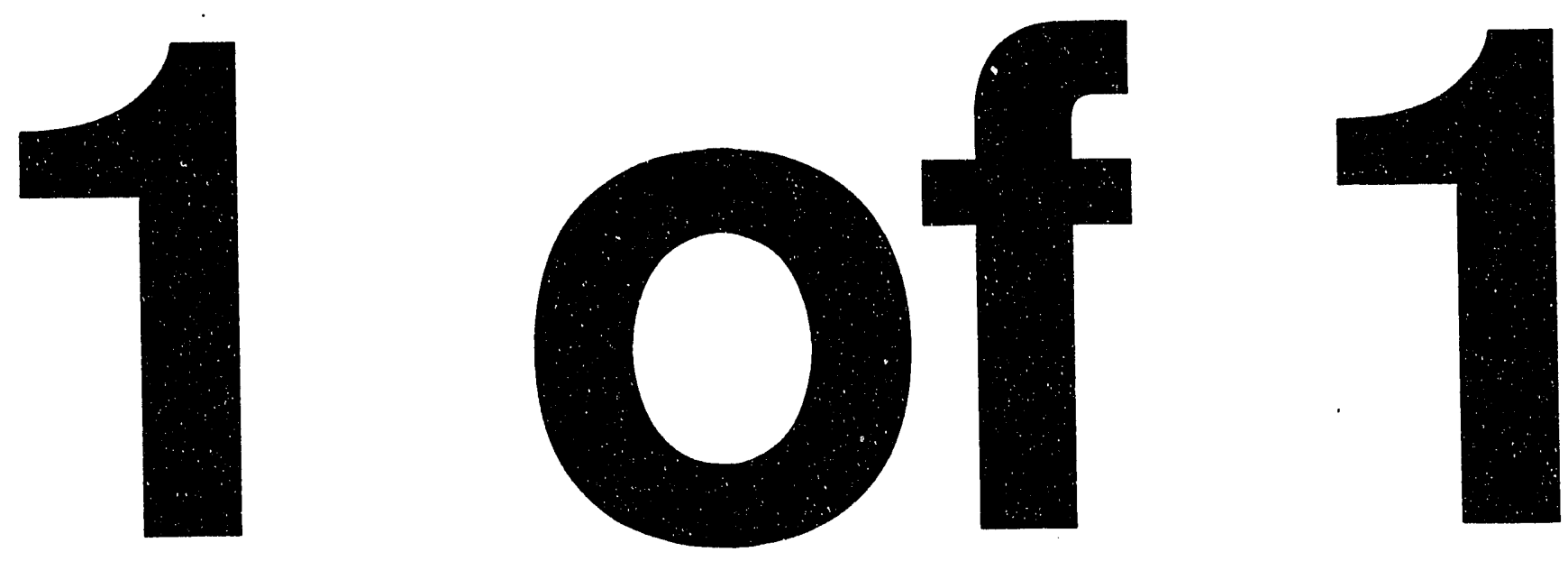


\title{
Conf- $9210246--19$
}

BNL- 48674

Informal Report

\section{An Interactive Beam Position Monitor System Simulator}

\author{
William A. Ryan \\ and \\ Thomas J. Shea
}

March 1993

\author{
RHICPROJECT \\ Brookhaven National Laboratory \\ Associated Universities, Inc. \\ Upton, New York 11973-5000
}




\section{DISCLAIMER}

This report was prepared as an account of work sponsored by an agency of the United States Government. Neither the United States Government nor any agency thereof, nor any of their employees, makes any warranty, express or implied, or assumes any legal liability or responsibility for the accuracy, completeness, or usefulness of any information, apparatus, product, or process disclosed, or represents that its use would not infringe privately owned rights. Reference herein to any specific commercial product, process, or service by trade name, trademark, manufacturer, or otherwise does not necessarily constitute or imply its endorsement, recommendation, or favoring by the United States Government or any agency thereof. The views and opinions of authors expressed herein do not necessarily state or reflect those of the United St ates Government or any agency thereof. 


\title{
AN INTERACTIVE BEAM POSITION MONITOR SYSTEM SIMULATOR*
}

\author{
W. A. Ryan and T. J. Shea \\ Brookhaven National Laboratory \\ Upton, NY 11973
}

\begin{abstract}
A system simulator has been implemented to aid the development of the RHIC position monitor system. Based on the LabVIEW software package by National Instruments, this simulator allows engineers and technicians to interactively explore the parameter space of a system during the design phase. Adjustable paraneters are divided into three categories: beam, pickup, and electronics. The simulator uses these parameters in simple formulas to produce results in both time-domain and frequencydomain. During the prototyping phase, these simulated results can be compared to test data acquired with the same software package. The RHIC position monitor system is presented as an example, but the software is applicable to several other systems as well.
\end{abstract}

\section{INTRODUCTION}

Simulation of the beam's interactions with beam position monitor (BPM) electrodes is a critical component of the EPM system design process. Originally the simulator described here simply calculated the response of a BPM electrode with programmable characteristics to a beam bunch or train of bunches also with programmable characteristics. The simulation was then extended to include cable and electronics response, as well as spectral analysis, position response, and linear aperture analysis. All programming was done in LabVIEW, an Macintosh-based analysis package that allows easy interactive manipulation of variables from the Panel window, with all graphical programming done in the Diagram window. These features are the key to the interactive nature of the simulator, as studies of many variable manipulations are easy to do, and programming modifications are quick and convenient to make.

\section{RHIC}

The RHIC storage rings ${ }^{[1],[2]}$ will have a rotation frequency of $78 \mathrm{kHz}$, and a bunching frequency of $4.45 \mathrm{MHz}$ with an upgrade to $8.9 \mathrm{MHz}$. Intensities will range from $10^{10}$ charges per bunch to $10^{11}$ charges per bunch. Bunch length will vary from 5.1 meters at injection to 1.2 meters at transition and storage. Most of the RHIC position monitors ${ }^{[3]}$ are pairs of $20 \mathrm{~cm}, 50 \mathrm{ohm}$ shorted striplines, each subtending an 80 degree azimuthal angle. The radius of the clear aperture is typically $38.1 \mathrm{~mm}$. At the

* Work períormed under the auspices of the US Department of Energy 
location of these BPMs, the dynamic aperture of the RHIC lattice is approximately \pm 20 $\mathrm{mm}$ in the plane of measurement (plane of maximum beta at the BPM location).

\section{LABVIEW AS A SIMULATION ENVIRONMENT}

Although LabVIEW ${ }^{[4]}$ was originally designed for data acquisition and analysis, many of its features, such as graphical programming, simple user interface, fast execution time and large quantities of available analysis routines make it convenient to use as an interactive simulator. All programs take the form of "Virtual Instruments"(VIs) that can either be run stand-alone or incorporated into other VIs. VIs are designed in two Macintosh windows: the Panel window containing the user interface, and the Diagram window containing the graphical source code. The numerical controls, numerical readouts and graphs are all viewed on the Panel window; programming is done in the Diagram window by interconnecting icons that represent VIs, controls, readouts and graphs.

Figure 1 contains the panel and diagram of a simple VI designed to generate a Johnson noise signal in the time domain.

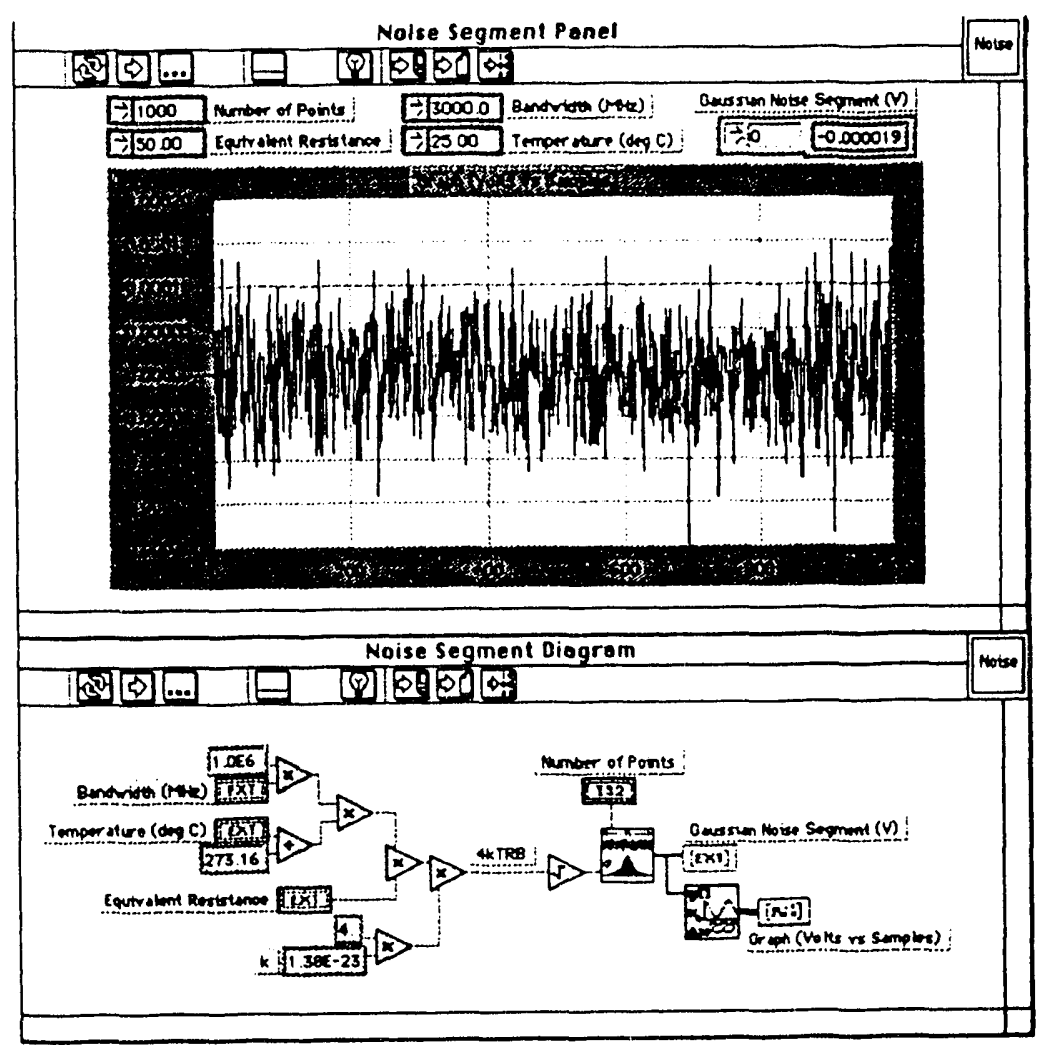

FIGURE 1. Noise Segment Virtual Instrument, Panel and Diagram, in LabVIEW

The panel contains four input variables: Number of Points, Equivalent Resistance, Bandwidth and Temperature. The graph and array output show the output noise segment. The diagram shows the actual source code, multiplying the proper inputs for the RMS noise voltage. The gaussian random signal is generated in another VI on the diagram, which can be opened to show its own source code. The Noise Segment VI itself can be used in this fashion, and its icon is shown in the upper right hand corner of the window. 


\section{TIME DOMAIN SIMULATION}

The simulator consists of three separate programs: Time Domain Analysis, Position Analysis, and Spectral Analysis. Because one goal of the RHIC BPM electronics development is effective single bunch position measurement, the main thrust of this simulator is single bunch response of the BPM and BPM electronics in the time domain.

The Time Domain Analysis determines the time-dependent response of the BPM, cable, detector, filters, and integrator to a single bunch or train of bunches. A representation of the program structure is shown in Figure 1.

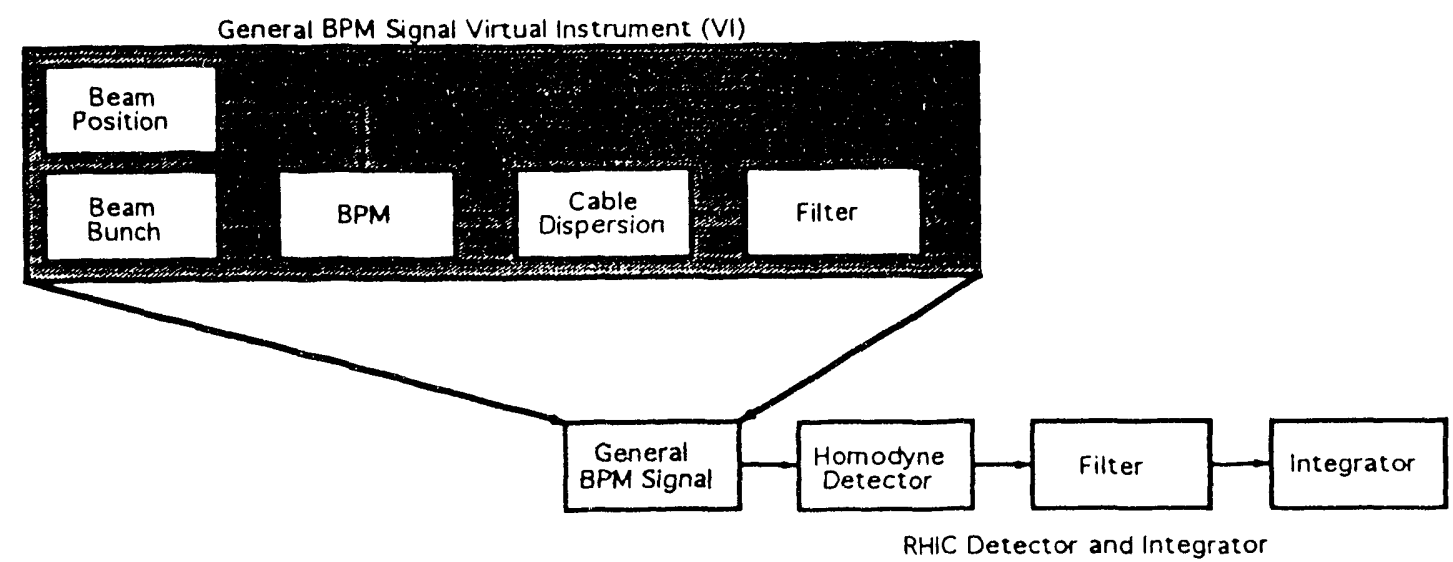

FIGURE 2. Single Bunch/Time Domain Simulator Structure

All boxes represent separate VIs, with internal programming, that can be accessed through icons in the overall Time Domain Analysis program structure. The Spectral Analysis and Position Response programs are similarly structured. Most formulas for position monitor response are adapted from Shafer ${ }^{[5]}$ and Lambertson ${ }^{[6]}$.

The Beam Bunch VI outputs an array representing the bunch current as described by the following expression:

$$
I_{\text {beam }}=\frac{2 c N e}{L} \cos ^{2}\left(\frac{\pi c t}{L}\right)
$$

where $\mathrm{Ne}$ is the charge in the bunch, $\mathrm{L}$ is the bunch length, and $\mathrm{c}$ is the speed of light.

The Beam Position VI calculates the fraction of the wall current seen by the left and right BPM striplines by summing a variable number of terms in the following cosine expansions:

$$
\begin{gathered}
I_{R}(t)=\left[\frac{-\phi I_{\text {beam }}(t)}{2 \pi}\right]\left[1+\left(\frac{4}{\phi}\right)\left\{\sum_{n=1}^{\infty} \frac{1}{n}\left(\frac{r}{b}\right)^{n} \cos (n \theta) \sin \left(\frac{n \phi}{2}\right)\right\}\right] \\
I_{L}(t)=\left[\frac{-\phi I_{\text {beam }}(t)}{2 \pi}\right]\left[1+\left(\frac{4}{\phi}\right)\left\{\sum_{n=1}^{\infty} \frac{1}{n}\left(\frac{r}{b}\right)^{n} \cos (n \theta) \sin \left(n\left(\phi+\frac{\pi}{2}\right)\right)\right\}\right]
\end{gathered}
$$

where $\emptyset$ is the subtended angle of the BPM and $\mathrm{r}$ and theta describe the beam position. The 
dipole term in these expansions can be used to obtain the linear part of the position monitor's response.

There are two types of detectors that can be selected to run the BPM VI: stripline and capacitive. The stripline detector has an added feature that all cable impedances and the stripline impedance can be set independently to study mismatch effects. For a perfectly matched system, the voltages at the position monitor ports can be obtained as follows:

$$
: V_{U}(t)=\left(\frac{\varphi Z}{4 \pi}\right)\left[I_{b}(t)-I_{b}\left(t-\frac{L}{\beta_{b} c}-\frac{L}{\beta_{s} c}\right)\right]
$$

for the upstream port and

$$
V_{D}(t)=\left(\frac{\varphi Z}{4 \pi}\right)\left[I_{b}\left(t-\frac{L}{\beta_{s} c}\right)-I_{b}\left(t-\frac{L}{\beta_{b} c}\right)\right]
$$

for the downstream port.

Capacitive electrode analysis is done by convolving the beam bunch current function with $(\varnothing \mathrm{L} / 2 \pi \mathrm{c})$ to give the charge w.r.t. time in the approximation of bunch length $>>$ electrode length. The charge per unit time is then multiplied by the capacitance and filtered which a single pole highpass formed by the electrode capacitance and the termination impedance.It should be noted that this simple model does not predict the high frequency resonances commonly observed in this type of pickup.

The cable dispersion is found by calculating, for an arbitrary length of cable, the impedance $^{[7]}$ :

$$
Z=j \omega\left(\frac{\mu_{0}}{2 \pi}\right) \operatorname{Ln}\left(\frac{r_{o}}{r_{i}}\right)+(1+j)\left(\frac{1}{2 \pi}\right)\left(\frac{1}{r_{o}}+\frac{1}{r_{i}}\right)\left(\frac{\omega \mu_{0} \rho}{2}\right)^{1 / 2}
$$

and the admittance:

$$
Y=\frac{j \omega 2 \pi \varepsilon \varepsilon_{0}}{\ln \left(r_{o} / r_{i}\right)}
$$

and then obtaining the propagation factor:

$$
\alpha+j k=(Y Z)^{1 / 2}
$$

which gives and phase shift and attenuation that are applied to the FFT of the input signal. The signal is then transformed back to the time domain with in inverse FFT. Both the FFT function and the IFFT are optimized VIs that come with the LabVIEW package.

In the simulation, the cable is followed by a choice of filter with programmable response. All filters are selectable from a collection of LabVIEW VIs that emulate a broad range of analog filters.

As shown in Figure 1, the structure of the time domain analysis allows the detector to be changed, while maintaining the BPM signals, to evaluate detector responses. The RHIC BPM Electronics currently under design consists of a bandpass filter followed by a homo- 
dyne detector, a lowpass filter, and an active integrator. A simplified schematic is shown in Figure 3.

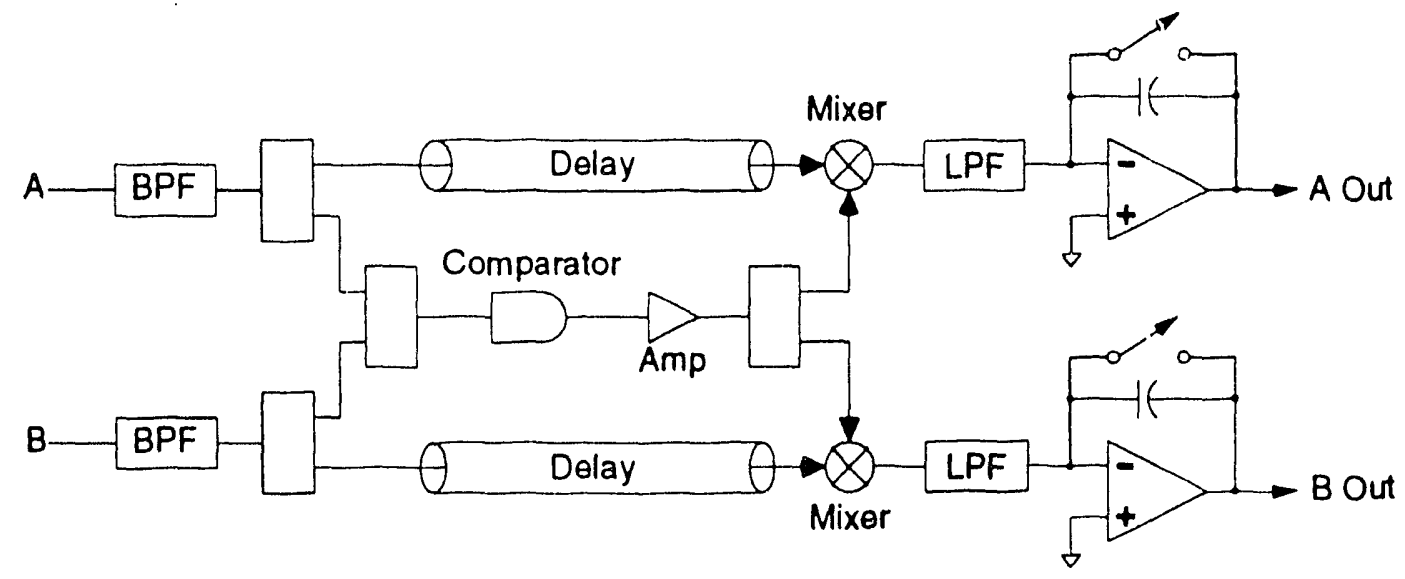

FIGURE 3. BPM Electronics Prototype - Block Diagram

\section{SPECTRAL ANALYSIS}

The spectral analysis simulator, a separate LabVIEW VI, numerically calculates the normalized Fourier spectrum of a cosine squared bunch of variable width and the frequency response of a stripline or capacitive BPM, and shows the product as the transmitted spectral amplitude response. The bunch spectrum is given by Equation 9 . By way of simple equivalent circuit approximations, the frequency responses of a stripline and a capacitive button are given by Equation 10 and Equation 11 respectively.

$$
\begin{gathered}
F_{\text {Bunch }}(\omega)=\left[\frac{2 c^{4} N e}{L^{3}}\right]\left[\frac{\sin \left(\frac{\omega L}{2 c}\right)}{\omega\left[\frac{4 \pi^{2} c^{2}}{L^{2}}-\omega^{2}\right]}\right] \\
F_{\text {Stripline }}(\omega)=\left(\frac{Z_{0}}{2}\right) \sin \left[\left(\frac{2 \pi L}{c}\right) f\right] \\
F_{\text {Capacitive }}(\omega)=\frac{(2 \pi R C) f}{\left(1+(2 \pi R C)^{2} f^{2}\right)^{1 / 2}}
\end{gathered}
$$

\section{POSITION RESPONSE}

Using Equation 2 and Equation 3, the position analysis simulator, also a separate LabVIEW VI, calculates the peak amplitude, total energy and average power for a single bunch linearly swept in position between given coordinate settings. The simulator also takes advantage of the expansion by isolating the dipole term and obtaining the linear response near the center of the BPM. Because coupling between the electrodes is ignored, the calculated sensitivity is naturally optimistic. The equations are the same as above. 
The position analysis algorithm has also been used to calculate the linear aperture of the RHIC BPM, not including edge effects. This is a comparison of the real position of a pencil beam. in the plane of measurement, with the measured position as a function of the orthogonal displacement from the axis of measurement. The difference between the real and measured positions is called off-axis error, and varies with the subtended angle of the electrode. For given beta functions at the BPM, an optimum subtended angle can be calculated.

\section{RESULTS}

The following are VIs and graphs generated on the RHIC BPM simulator.

Figure 1 shows the Panel window, with graphs and variable controls, for the Time Domain Simulator. The graph currently shows the signal output of a BPM in response to a $5.1 \mathrm{~m}$ long bunch of $10^{1 \mathrm{l}}$ protons. The controls are shown in blocks designating which VI they address. The beam bunch characteristics control contains a switch allowing the number of points to be forced to a power of two, which allows the cable analysis to use a FFT algorithm instead of a DFT algorithm and dramatically decreases run time. The BPM controls allow either a stripline or capacitive BPM to be simulated, with arbitrary terminations. As the RHIC BPM is a one-port shorted stripline, this is a special case in the BPM Type control. In the position VI, the position can be input in either polar or cartesian form. The filter control allows any type or characteristic analog filter to be specified. Signals at any point in this chain, along with peak amplitude, energy and average power calculations, can be viewed in the Array Graph by toggling the controls on left side of the graph.

Figure 1 contains a normalized graph of the bunch spectrum, calculated analytically from the bunch length assuming a cosine squared bunch profile, together with the stripline BPM response, calculated from the stripline length (capacitive electrodes can also be simulated). Also shown is the product of the two, representing the transmitted spectral power.

Figure 1 shows the position analysis VI, simulating the response to a pencil beam swept over the indices specified in the upper right hand corner. Centered signal amplitude and power is specified elsewhere. The VI will show the response of both electrodes, the sum, difference, and $\Delta \sqrt{\Sigma}$, along with the $\log$ ratio, $\log$ ratio in $\mathrm{dBs}$, and measured position (using $\log$ ratio) vs. real position. Units can be peak amplitude, energy, or average power.

Figure 1 is a graph relating to the linear aperture of the RHIC BPM, calculated using a VI related to the position analysis VI. This graph shows the major axis of the maximum allowable ellipse that encompasses certain values of off-axis error, graphed as a function of stripline subtended angle. The ellipticity is equal to the square root of the ratio of the beta functions at the BPM location. The ellipse major axis is measured in centimeters, while the off-axis error is displayed as a fraction of the beam pipe radius. 


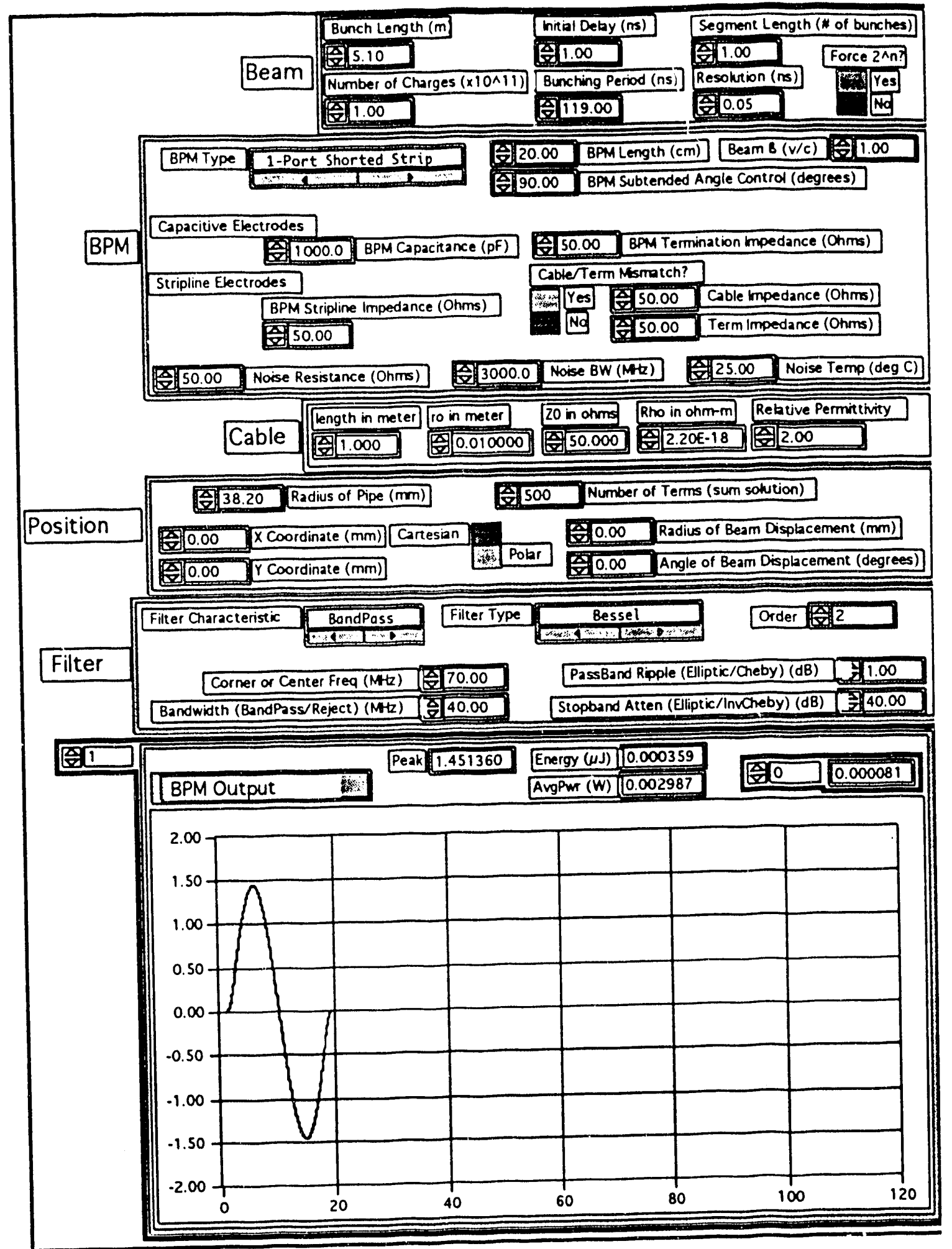

FIGURE 4. Panel of General BPM Signal VI, with RHIC BPM output graph for maximum bunch length, $5.1 \mathrm{~m}$ at injection. 


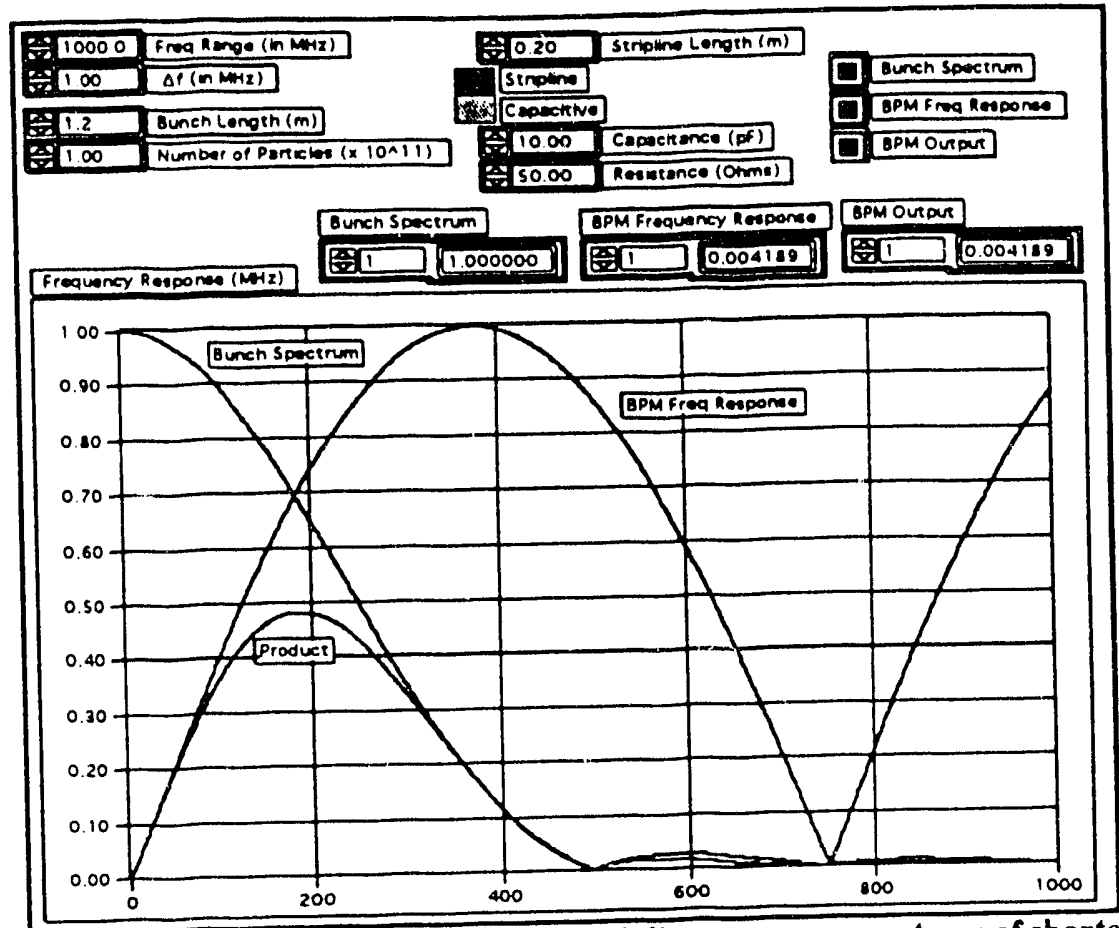

FIGURE 5. Normalized graph of $20 \mathrm{~cm}$ shorted stripline response, spectrum of shortest RHIC single bunch $(1.2 \mathrm{~m})$, and product.

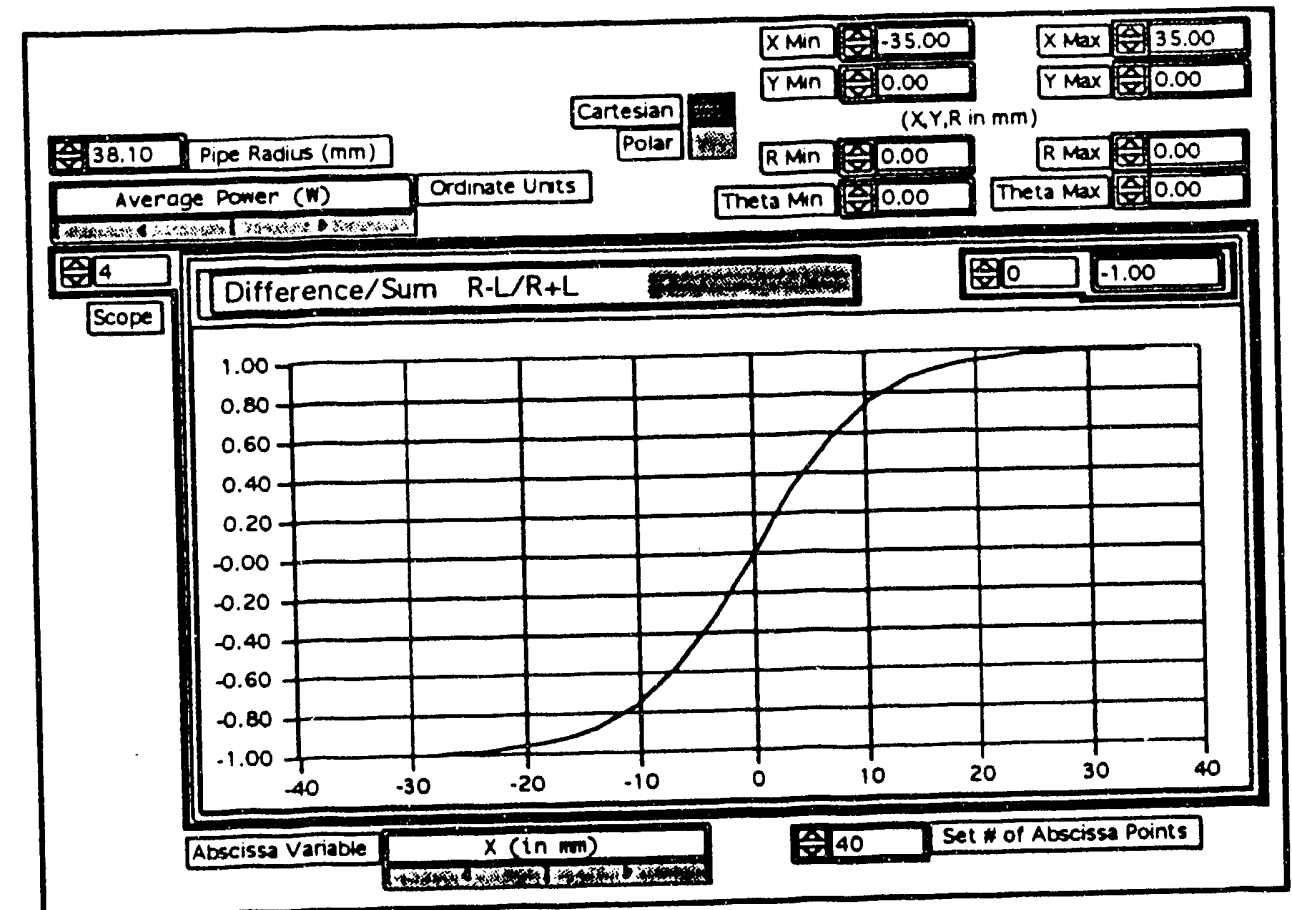

FIGURE 6. Difference over sum processing of average power response, sweeping pencil beam $+1-35 \mathrm{~mm}$ in plane of measurement (X axis). 


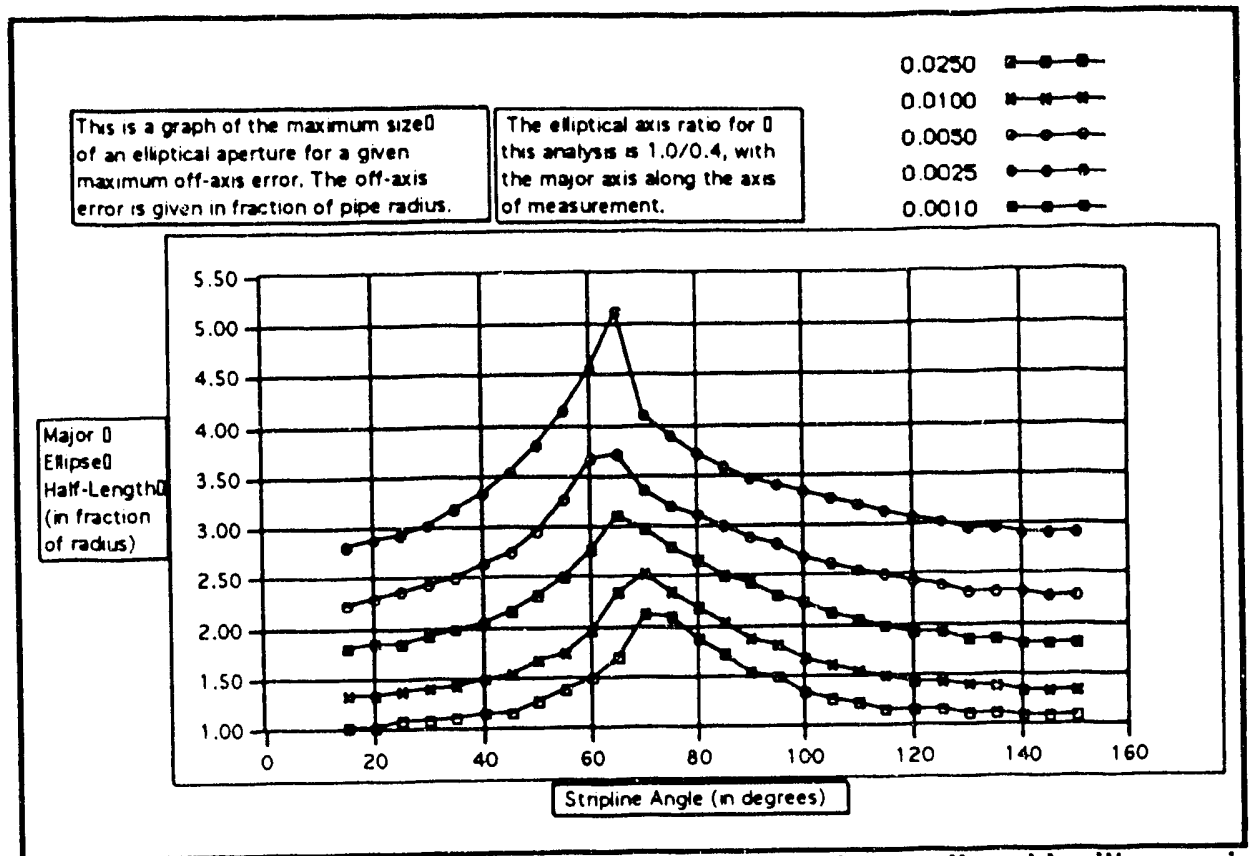

FIGURE 7. Result of off-axis error analysis. Graph of maximum allowable ellipse major axis (in RHIC the major/minor axis ratio $=1: 0.4$ ) for given value of off-axis error, vs. subtended angle of RHIC stripline. (Major Axis length is in $\mathrm{cm}$, Error is in fraction of pipe radius)

\section{ACKNOWLEDGEMENTS}

Acknowledgment is given to Vibha Mane for writing the cable dispersion software, and to the RHIC Beam Instrumentation Group for significant discussions.

\section{REFERENCES}

1. Satoshi Ozaki, "RHIC Project," Conference Record of the 1991 IEEE Particle Accelerator Conference, IEEE-91CH3038-7, San Francisco (May 1991).

2. Conceptual Design of the Relativistic Heavy Ion Collider (RHIC), Brookhaven National Laboratory, Associated Universities, Inc., BNL 52195 UC-414 (1991).

3. T. J. Shea, "Design Studies on the RHIC Beam Position Monitor System," BNL Informal Report, BNL-48048, AD/RHIC-114 (1992).

4. LabVIEW ${ }^{\circledR} 2$ User Manual, September 1991 Edition, Part Number 320244-01, National Instruments, Inc., Austin TX.

5. R.E.Shafer, "Characteristics of Directional Coupler Beam Position Monitors," IEEE Transactions on Nuclear Science, Vol. NS-32, No.5 (October 1985).

6. G. Lambertson, "Dynamic Devices; Pickups and Kickers," AIP Conf. Proc. 153, M. Month, Ed., 1413 (1987)

7. Simon Ramo, John R. Whinnery, Theodore van Duzer, Fields and Waves in Communication Electronics, Second Edition, John Wiley \& Sons, New York (1984). 

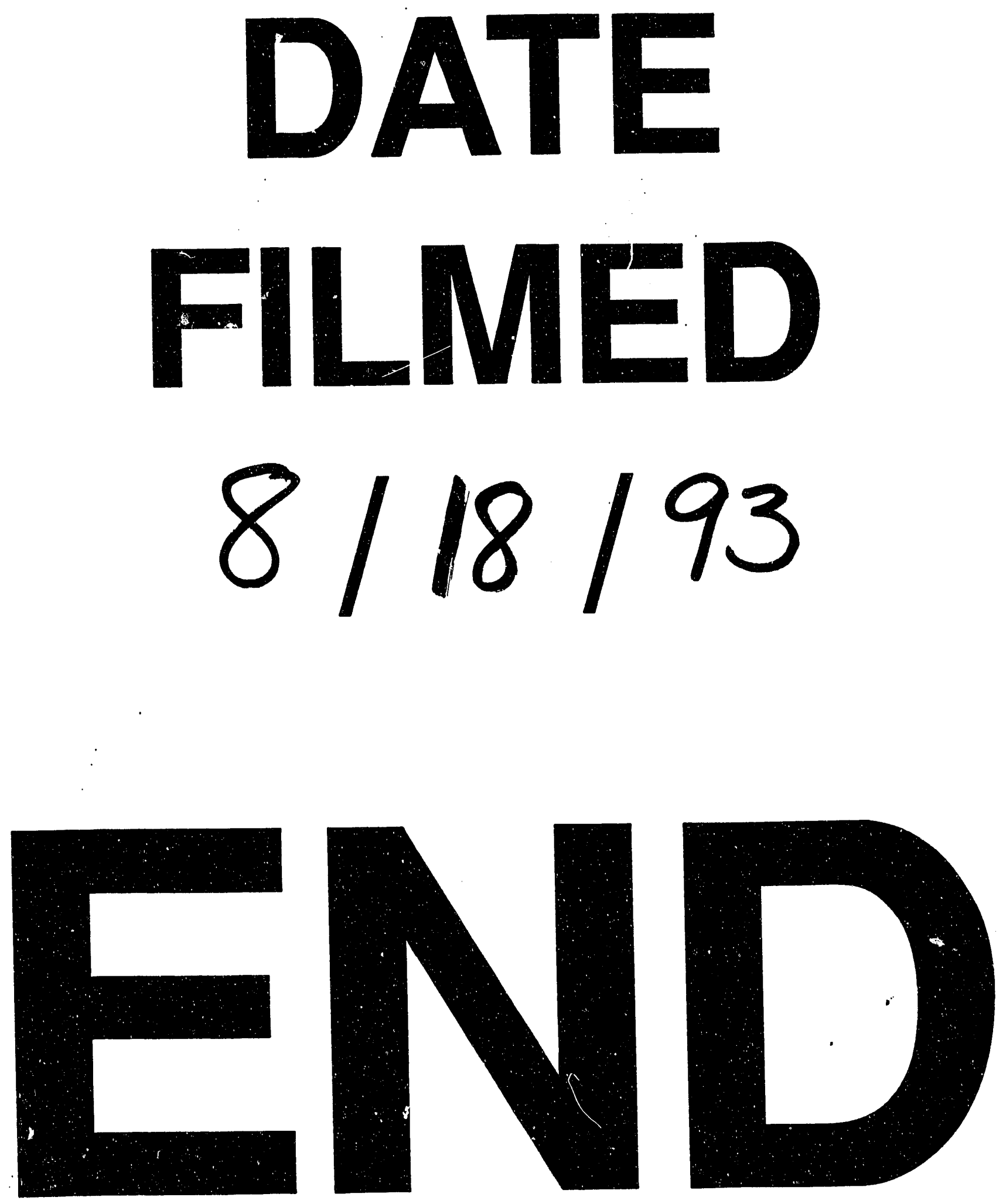
